\title{
U-40 TRIFFT Ü-50 - WISSENSAUSTAUSCH ZWISCHEN DEN GENERATIONEN
}

\section{Liebe Kolleginnen und Kollegen,}

nach 6 Jahren Einsatz für unsere Vereinszeitschrift wird unsere geschätzte Kollegin Frau Rita Klowersa die Schriftleitung an ein neues Team übergeben. Herr Reis wird den Bereich Körperakupunktur übernehmen, Frau Strittmatter den Bereich Ohrakupunktur und Frau Beyersdorf wird für das Projektmanagement verantwortlich sein. Wir bedanken uns bei Frau Klowersa für die vielen Hefte mit interessanten Inhalten. Da ihr die Gestaltung der Titelseite immer ein besonderes Anliegen war, veröffentlichen wir in Anerkennung dieser Arbeit in dieser Ausgabe eine Collage einzelner Titelbilder.

Die Einführungskurse sind gut angelaufen und das neue Ohrraster wird von den Teilnehmern sehr gut angenommen. F. Bahr wird im Laufe des Jahres nach und nach die einzelnen Koordinaten in der Mitgliederzeitschrift veröffentlichen. Freuen wir uns in diesem Heft auf die Lokalisationsangaben im Cavum conchae. Der Internationale Kongress (WECAM) wurde von Wien 2016 nach Singapur 2017 (10.-12.08.2017) verlegt, sodass wir mit dem neuen Ohrraster genügend Erfahrung sammeln können, um die internationale Akzeptanz für diese Nomenklatur $\mathrm{zu}$ erreichen.

Dank Ihrer Mitgliedsbeiträge konnten wir zu Beginn des Jahres wieder Forschungsgelder für die RAC-Forschung zur Verfügung stellen. Auf dem Akupunkturkongress am Timmendorfer Strand (05.-09.09.2016) erwartet uns ein Vortrag von G. Litscher über Innovationen und selektive Trends in der Entwicklung der Ohrakupunkturforschung.

In diesem Jahr wollen wir mit verschiedenen Aktionen den Nachwuchs in der Ganzheitsmedizin fördern. Dazu wird es erstmalig eine Aktion „U-40 trifft Ü-50“ beim Akupunkturkongress am Timmendorfer Strand geben. Erfahrene Akupunkteure über 50 Jahre erhalten ebenso wie junge KollegInnen, die sie für den Besuch des Kon- gresses gewinnen konnten, einen Rabatt von $25 \%$ auf die Kongressgebühr.Was wäre die Erfahrungsheilkunde ohne den Wissensaustausch zwischen den Generationen? Wir hoffen, dass sich auf diese Weise auch ein Mentoren-Schüler-Verhältnis entwickelt, das über den Kongress hinaus den jungen Akupunkteuren eine Hilfe ist. Bitte beachten Sie die Einladung zum Akupunkturkongress, der konkrete Informationen zum Ablauf dieser Aktion beiliegen.

Die Deutsche Akademie für Akupunktur Berufsverband (DAA-B) informiert:

GOÄ aktuell vom Tisch, aber die Probleme bleiben (Analogziffer, Steigerungsfaktoren, Ausnahmeregelungen).

Am 10.05.16 wurde in einer Telefonkonferenz ein Konsens der Akupunktur-Fachgesellschaften zu diesem Thema erreicht. Die Belange der Akupunkturärzte in Deutschland werden der BÄK vorgetragen und finden hoffentlich in einer überarbeiteten Version ihre Berücksichtigung. Wesentliche Forderungen betreffen „Neue Gebührenordnungspositionen " - hier insbesondere die Pulsdiagnostik (traditionelle und moderne Verfahren wie der NogierReflex) und „Zuschläge“ zu bestehenden GOP. Ende Juli wird bei der BÄK unter Teilnahme der Fachverbände die Abstimmung der Kapitel „Grundleistungen, Allgemeinmedizin“" sowie „Nichtgebietsbezogene Sonderleistungen" stattfinden.

Mit guten Wünschen für den Sommer

Ihr

B. Ramme

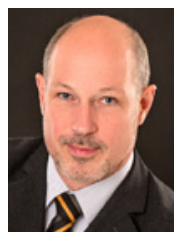

Dr. med. Bernd Ramme

1. Vorsitzender der DAA e.V.,

Osserstraße 40, D-81679 München

Tel. $+4989 / 8145252$

E-Mail des allg.Büros: kontakt@akupunktur.de, Internet:www.akupunktur.de 